\title{
Qualitative and Quantitative Comparison of PET/CT and PET/MR Imaging in Clinical Practice
}

\author{
Khalsa Z. Al-Nabhani, Rizwan Syed, Sofia Michopoulou, Jokha Alkalbani, Asim Afaq, Emmanouil Panagiotidis, \\ Celia O'Meara, Ashley Groves, Peter Ell, and Jamshed Bomanji \\ Institute of Nuclear Medicine, University College London Hospital NHS Foundation Trust, London NW1 2BU, United Kingdom
}

The aim of this study was to prospectively compare whole-body $\mathrm{PET} / \mathrm{MR}$ imaging and PET/CT, qualitatively and quantitatively, in oncologic patients and assess the confidence and degree of inter- and intraobserver agreement in anatomic lesion localization. Methods: Fifty patients referred for staging with known cancers underwent PET/CT with low-dose CT for attenuation correction immediately followed by PET/MR imaging with 2-point Dixon attenuation correction. PET/CT scans were obtained according to standard protocols $(56 \pm 20 \mathrm{~min}$ after injection of an average 367 $\mathrm{MBq}$ of ${ }^{18} \mathrm{~F}-\mathrm{FDG}, 150 \mathrm{MBq}$ of ${ }^{68} \mathrm{Ga}$-DOTATATE, or $333.8 \mathrm{MBq}$ of ${ }^{18} \mathrm{~F}$-fluoro-ethyl-choline; $2.5 \mathrm{~min} /$ bed position). PET/MR was performed with $5 \mathrm{~min} /$ bed position. Three dual-accredited nuclear medicine physicians/radiologists identified the lesions and assigned each to an exact anatomic location. The image quality, alignment, and confidence in anatomic localization of lesions were scored on a scale of 1-3 for PET/CT and PET/MR imaging. Quantitative analysis was performed by comparing the standardized uptake values. Intraclass correlation coefficients and the Wilcoxon signed-rank test were used to assess intra- and interobserver agreement in image quality, alignment, and confidence in lesion localization for the 2 modalities. Results: Two hundred twenty-seven tracer-avid lesions were identified in 50 patients. Of these, 225 were correctly identified on PET/CT and 227 on PET/MR imaging by all 3 observers. The confidence in anatomic localization improved by $5.1 \%$ when using PET/MR imaging, compared with PET/CT. The mean percentage interobserver agreement was $96 \%$ for PET/CT and $99 \%$ for PET/MR imaging, and intraobserver agreement in lesion localization across the 2 modalities was 93\%. There was $10 \%(5 / 50$ patients) improvement in local staging with PET/MR imaging, compared with PET/CT. Conclusion: In this first study, we show the effectiveness of whole-body PET/MR imaging in oncology. There is no statistically significant difference between PET/MR imaging and PET/CT in respect of confidence and degree of inter- and intraobserver agreement in anatomic lesion localization. The PET data on both modalities were similar; however, the observed superior soft-tissue resolution of MR imaging in head and neck, pelvis, and colorectal cancers and of CT in lung and mediastinal nodal disease points to future tailored use in these locations.

Received Mar. 22, 2013; revision accepted Jul. 31, 2013.

For correspondence or reprints contact: Rizwan Syed, Institute of Nuclear Medicine, University College Hospital, 235 Euston Rd., London NW1 2BU, U.K.

E-mail: rizwan.syed@uclh.nhs.uk

Published online Dec. 12, 2013.

COPYRIGHT (C 2014 by the Society of Nuclear Medicine and Molecular Imaging, Inc.
Key Words: PET/CT; PET/MR imaging; cancer; intraobserver agreement

J Nucl Med 2014; 55:88-94

DOI: $10.2967 /$ jnumed.113.123547

$\mathbf{T}$

he development of any new imaging technology generates significant interest. The introduction of PET with ${ }^{18} \mathrm{~F}$ FDG was an important step in metabolic imaging; however, the restricted accuracy of anatomic localization of lesions with PET alone was a significant limitation (1).

The introduction of combined PET/CT imaging in 1998 was a major breakthrough in hybrid imaging technology (2), and it is now an important staging modality in oncology patients (3-6). Similarly, the recent introduction of truly integrated PET/MR imaging scanners has generated much interest. The superior softtissue contrast of MR imaging, compared with CT, in evaluating lesion infiltration in soft-tissue planes and adjacent organs is a major step forward in the staging of various cancers $(7,8)$, particularly in the brain, head and neck, liver, pelvis, and bone marrow $(8,9)$. Furthermore, the lack of radiation using MR imaging is a major advantage over CT, though this is less significant after the introduction of CT dose modulation software, which has reduced the radiation to $2-5 \mathrm{mSv}$ without any compromise in resolution $(10,11)$. Nevertheless the benefit from PET/MR over PET/CT remains significant from a radiation exposure point of view in pediatric applications and in the many repeated studies inserted in patient management pathways.

The aim of this study was therefore to compare PET/MR images and PET/CT images qualitatively and quantitatively in oncologic patients and assess the confidence and degree of interobserver and intraobserver agreement in anatomic lesion localization.

\section{MATERIALS AND METHODS}

\section{Patient Population}

This was a prospective study approved by the local ethics committee, and all patients gave written informed consent. Patient inclusion criteria were as follows: informed consent, histologically confirmed cancer, and ability to undergo 2 scanning procedures. The exclusion criteria were pregnant women and claustrophobic patients or patients with cardiac pacemaker, metallic implants, cochlear implants, and permanent contraceptive devices. The PET/CT and PET/MR machines were housed in different parts of the hospital complex. The travel time between the 2 machines varied from patient to patient (range, 10-45 min), depending on their mobility (walking, wheelchair, or bed bound). 


\section{Hardware}

The Biograph mMR (Siemens) was used for PET/MR studies and Discovery VCT (GE Healthcare) for PET/CT. The 2 scanners have different PET detector technologies. Specifically, the PET component of the Biograph mMR uses smaller-sized lutetium orthosilicate detectors and has a longer axial field of view (FOV), providing higher sensitivity and slightly better spatial resolution than the GE Discovery VCT, which uses larger bismuth germanate crystals. Differences in scanner technology and data reconstruction methods can potentially affect image quality and standardized uptake value (SUV) quantification, and we routinely apply standardized methods to minimize such differences (12-13). In this study, the reconstruction parameters between the 2 systems were matched; however, differences inherent to the individual manufacturer's algorithms cannot be completely eliminated.

$P E T / C T$. All patients underwent whole-body PET/CT from the vertex to the mid thigh after a single intravenous injection of PET tracers ${ }^{18} \mathrm{~F}$-FDG, ${ }^{68} \mathrm{Ga}$-DOTATATE, or ${ }^{18} \mathrm{~F}$-fluoro-ethyl-choline $\left({ }^{18} \mathrm{~F}\right.$ $\mathrm{FECH}$ ), according to a standard clinical protocol performed on an integrated 64-slice PET/CT scanner (Discovery VCT; GE Healthcare). Acquisition began at $60 \mathrm{~min}$ after injection of ${ }^{18} \mathrm{~F}-\mathrm{FDG}(89-482 \mathrm{MBq}$; average activity, $367.69 \mathrm{MBq}$ ), $45-50 \mathrm{~min}$ after ${ }^{68} \mathrm{Ga}$-DOTATATE $(138-180 \mathrm{MBq}$; average activity, $150 \mathrm{MBq})$, and $60 \mathrm{~min}$ after ${ }^{18} \mathrm{~F}$ FECH (198-410 MBq; average activity, 327.4 MBq). For pediatric patients, the doses were adjusted according to weight. For ${ }^{18} \mathrm{~F}-\mathrm{FDG}$ studies, patients fasted for $4-6 \mathrm{~h}$, and the cutoff for fasting blood glucose was $10 \mathrm{mmol} / \mathrm{L}$.

Different tracers were used for different types of malignancy. ${ }^{18} \mathrm{~F}-$ FDG, which is the ${ }^{18} \mathrm{~F}$-labeled form of 2-deoxyglucose, is commonly used for imaging most cancers. ${ }^{68} \mathrm{Ga}$-DOTATATE targets somatostatin type II receptors and was used for detection of neuroendocrine tumors. ${ }^{18} \mathrm{~F}-\mathrm{FECH}$ is the fluorinated choline analog. Choline is an essential nutrient involved in the synthesis of the phospholipid components of cell membranes. Here, ${ }^{18} \mathrm{~F}-\mathrm{FECH}$ was used mainly for the evaluation of prostate cancer and brain tumors. Of 50 patients, 34 (68\%) underwent an ${ }^{18} \mathrm{~F}$-FDG scan, $12(24 \%)$ an ${ }^{18} \mathrm{~F}-\mathrm{FECH}$ scan, and $4(8 \%)$ a ${ }^{68} \mathrm{Ga}$-DOTATATE scan.

A nonenhanced low-dose CT scan was acquired for attenuation correction at $120 \mathrm{keV}$ with $10 \mathrm{~mA}$ (couch movement of $0.8 \mathrm{~s}$ and $30 \mathrm{~mm}$ per rotation). Attenuation-corrected transaxial slices $(128 \times 128$ matrices, corresponding to a 70-cm-diameter FOV) were reconstructed using the standard software of the scanner (3 dimensions; iterative reconstruction; heavy filter; postprocessing filter, $5.14 \mathrm{~mm}$; 21 subsets; 2 iterations). With a bilinear transformation based on the use of different scaling factors for materials with Hounsfield units, the CT images were converted to maps of PET attenuation coefficients. PET images were reconstructed using CT attenuation maps. PET acquisitions were acquired in 3 dimensions with 11-slice overlap and were modulated according to patient body mass index: $<25$, $2.5 \mathrm{~min} /$ bed position; $25-30,3 \mathrm{~min} / \mathrm{bed}$ position; $30-35,4 \mathrm{~min} / \mathrm{bed}$ position; and $>35,5 \mathrm{~min} /$ bed position.

PET/MR Imaging. PET/MR imaging was performed using a Siemens 3T Biograph mMR system with an integrated PET system within the MR gantry, which allows simultaneous PET and MR acquisitions without having to reposition the patient. The total examination time varied between $20 \mathrm{~min}$ for a 3-bed position acquisition and $35 \mathrm{~min}$ for a 5-bed position acquisition.

The PET/MR imaging scan was started $135 \pm 36$ min after injection. Acquisition was caudocranial, with multiple bed positions. The first MR sequence undertaken at each bed position was a coronal 2-point Dixon sequence for MR attenuation-correction purposes. A 3-dimensional volumetric interpolated breath-hold examination T1-weighted MR imaging sequence was acquired (integrated parallel acquisition techniques [iPAT] factor, 2 ; voxel size, $4.1 \times 2.6 \times 3.1 \mathrm{~mm}$ [in-plane resolution, slice thickness]; acquisition time, $19 \mathrm{~s}$; repetition time, $3.6 \mathrm{~ms}$; first echo time, $1.225 \mathrm{~ms}$; second echo time, $2.45 \mathrm{~ms}$; matrix, $79 \times 192$; number of excitations, 1; FOV, 500 mm; phase FOV, 65.5\%; 1 slab with 128 slices; slice thickness, $3.1 \mathrm{~mm}$; flip angle, 10; and bandwidth, 960 $\mathrm{Hz} /$ pixel). Centric k-space acquisition was used to minimize artifacts from respiratory movements.

During the time taken for the free-breathing PET acquisition, PET was acquired statically for $5 \mathrm{~min}$ in each bed position. In addition, an axial T2-weighted imaging half Fourier acquisition single-shot turbo spin echo (T2WI HASTE) sequence (repetition time, 1,600 ms; echo time, $100 \mathrm{~ms}$; FOV, $376 \times 501$; 5-mm slice thickness; 36 slices) and freebreathing axial 3-directional echo planar imaging diffusion data at $3 \mathrm{~b}$ values $\left(0,400\right.$, and $800 \mathrm{~s} / \mathrm{mm}^{2}$; repetition time, $8,700 \mathrm{~ms}$; echo time, 88 ms; 2 averages; FOV, $282 \times 350$; 5 -mm slice thickness; 30 slices; short $\mathrm{T} 1$ inversion recovery fat suppression) were acquired. These datasets were used to create apparent diffusion coefficient maps with Siemens software (version Syngo MR B18P). The PET data were reconstructed using ordered-subset expectation maximization with 21 subsets and 3 iterations and a gaussian filter of $5 \mathrm{~mm}$ in full width at half maximum (FOV, $359 \times 359 \mathrm{~mm}$; 2-mm slice thickness; 127 slices).

An axial T1 volumetric interpolated breath-hold examination 2-point Dixon sequence was acquired (iPAT factor, 2; voxel size, $1.6 \times 1.2 \times$ $4.0 \mathrm{~mm}$ [in-plane resolution, slice thickness]; acquisition time, $17 \mathrm{~s}$; repetition time, $4.13 \mathrm{~ms}$; first echo time, $1.31 \mathrm{~ms}$; second echo time, $2.54 \mathrm{~ms}$; matrix, $188 \times 320$; number of excitations, 1 ; FOV, $380 \mathrm{~mm}$; phase FOV, 78.1\%; 1 slab with 64 slices; slice thickness, $3.0 \mathrm{~mm}$; flip angle, 9; and bandwidth, 1,040 Hz/pixel]).

The Dixon fat- and water-weighted images were used to create an attenuation map with 4 distinct tissue classes: background, lungs, fat, and soft tissue $(14,15)$. Connected-component analysis of the air in the inner part of the body was used to correct for lung attenuation. A morphologic closing filter was used for correction of virtual air artifacts in the pelvis, aorta, bones, and vessels. There are some known limitations of MR-based attenuation-correction methods. Specifically, the Dixon-based maps account for soft tissue, fat, and lung correction but disregard bone tissue, thus underestimating the SUV for lesions within or near bony structures $(14,15)$. This effect was observed in ${ }^{18} \mathrm{~F}$-FECH PET/MR studies for brain tumors, where the SUVs were underestimated and attributed to the fact that the skull is not considered when using the 2-point Dixon MRI-based AC (magnetic resonance imaging-based attenuation correction). Recently, we started using an ultrashort time echo sequence, which detects signal from cortical bone (16). Further limitations of MR-based attenuation-correction methods include truncation of the arms and the lack of correction for the flexible-body RF coils, which are, however, designed using low-attenuation materials (17).

\section{Data Analysis}

In all cases, PET/CT was performed before PET/MR imaging. Three dual-accredited nuclear medicine physicians/radiologists with 10,5 , and $4 \mathrm{y}$ of experience in reporting CT, PET/CT, and MR imaging studies analyzed all the examinations on dedicated Siemens Syngo (PET/MR imaging) and GE Healthcare Advantage (PET/CT) workstations. The observers were aware that they were evaluating studies for patients with cancers and possible metastases, but were masked to the radiologic findings.

They were given anonymized PET/MR and PET/CT studies at random with a gap of $4 \mathrm{wk}$ between the 2 scans for interpretation. This was done to reduce the recollection of imaging findings. The observers interpreted visually only the low-dose CT, PET/CT, T1-weighted (T1WI) and T2WI HASTE MR, diffusion-weighted imaging (DWI)/ $\mathrm{ADC}$, and PET/MR images without any clinical information to identify and anatomically localize the lesions and to accurately stage the disease. A 2-point Dixon sequence was used for attenuation correction only. Discrepant cases were resolved by consensus. 


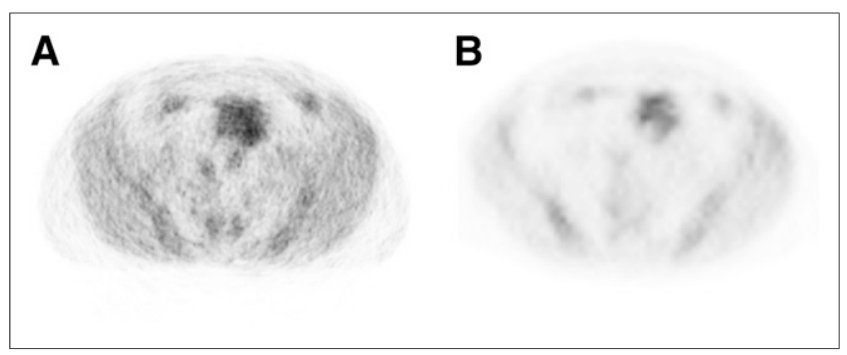

FIGURE 1. A 54-y-old man with lymphoma. (A) Subtle pattern of concentric rings noticeable on attenuation-corrected PET image of PET/MR. (B) Same section on attenuation-corrected PET component of PET/CT.

Subjective analysis of the image quality of the 2 modalities was scored on a scale of 1-3 (unacceptable, 1; good, 2; and excellent, 3). The alignment between PET and CT images and between PET and MR images was scored using a scale of 1-3 (no misalignment, 1; minor misalignment, 2; and major misalignment, 3) based on the accurate registration of fused images at the interface of the right hemi-diaphragm and dome of the liver. The observers were requested to identify the lesions and to assign each to an exact anatomic location on both lowdose CT and T1WI and T2WI HASTE MR images. The confidence in anatomic localization of lesions was scored on a scale of 1-3 on PET/CT and PET/MR imaging (exact anatomic region unknown, 1; probable site of anatomic region, 2; and definite anatomic localization, 3 ).

Quantitative analysis was performed by comparing the SUVs derived by PET/CT and PET/MR imaging. Specifically, $3 \mathrm{~mm}^{3}$ regions of interest over the lesion and selected background areas corresponding to the brain, mediastinum, liver, and soft tissue were free handdrawn. The mean and maximum SUVs of each region of interest were recorded on both modalities.

\section{Clinical Follow-up}

All patients who underwent PET/CT and PET/MR imaging were discussed in a multidisciplinary team meeting, and management decisions were documented. The multidisciplinary team clinical notes were reviewed later to ascertain the management change after discussion of PET/CT and PET/MR imaging findings.

\section{Statistics}

The concordance between the lesion localization score provided by the 3 observers was expressed in terms of mean percentage agreement. Intraclass correlation coefficients were used to assess the intraobserver agreement in confidence of lesion localization across the 2 modalities and also interobserver agreement within the same modality. The Wilcoxon signed-rank test was used to assess statistical differences in lesion localization, image quality, and alignment between the 2 modalities. SUV results were evaluated by nonparametric correlation analysis, using the Spearman rank correlation coefficient to examine the association between mean SUVs derived from the 2 modalities. The Wilcoxon signed-rank test was used to assess statistical differences between these values. In all cases, statistical significance was accepted for $P$ values lower than 0.01 .

\section{RESULTS}

Between March and November 2012, 70 consecutive patients with histologically proven malignancy and possible metastases referred for PET/CT were recruited for this cross-over PET/MR imaging study. The study included a wide spectrum of oncologic patients. All patients gave informed consent and initially underwent their clinically indicated PET/CT scan $\left({ }^{18} \mathrm{~F}-\mathrm{FDG}\right.$, ${ }^{68} \mathrm{Ga}$-DOTATATE, or ${ }^{18} \mathrm{~F}$-FECH), followed by simultaneous whole-body PET/MR imaging examination, on the same day. Of
TABLE 1

Results of Image Quality Grading

\begin{tabular}{cccc}
\hline Modality & Unacceptable & Good & Excellent \\
\hline PET/MR & 0 & 39 & 11 \\
PET/CT & 0 & 38 & 12
\end{tabular}

Wilcoxon rank test, $P=0.768$.

the 70 patients, 50 completed the cross-over study (15 women, 35 men; age range, 11-76 y; median, 50.2 y) (Supplemental Table 1; supplemental materials are available at http://jnm.snmjournals. org). Twenty cases were excluded, because of equipment failure ( 6 patients), prolonged time interval $(>3 \mathrm{~h}$ ) between PET/CT and PET/MR imaging ( 7 patients), or inability of the patient to continue PET/MR imaging due to claustrophobia or anxiety and pain (7 patients). The scan was obtained for local staging of disease $(n=$ $14)$, planning of treatment $(n=13)$, monitoring response to treatment $(n=21)$, or differentiation between recurrent disease and posttherapy changes $(n=2)$.

In the 50 patients who completed the study, the MR imaging datasets acquired during simultaneous PET acquisition showed diagnostic image quality without any significant artifacts or distortions related to the PET component. The PET images from the PET/MR imaging scanner showed a subtle pattern of concentric rings, compared with conventional PET/CT (Fig. 1). There was no significant difference in the image quality of the non-attenuation-corrected images between the 2 modalities. Attenuation-correction image artifacts were noticeable on both modalities secondary to implants (4 portacaths, 3 central venous lines, and 4 prostheses) and liver hemosiderosis $(n=1)$, which were not detectable on non-attenuation-corrected images. The qualitative grading results summarized in Table 1 showed only marginal differences between the PET/CT and PET/MR image quality, which were not statistically significant $(P=0.768)$. There

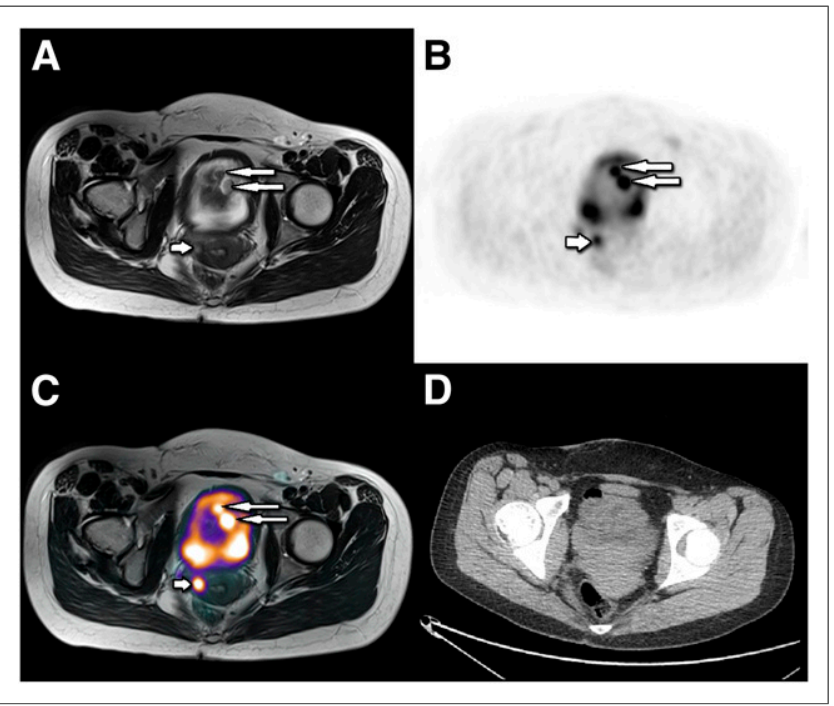

FIGURE 2. (A-D) A 16-y-old woman with recurrent multiple vaginal paragangliomas. (A-C) Fused images of pelvis, respectively, show tracer-avid hyperintense lesions at dome of bladder (long arrows) and right lateral upper vaginal wall (short arrows). (D) Axial nonenhanced CT image of pelvis at level of bladder does not show any obvious abnormality along superior aspect of bladder. 


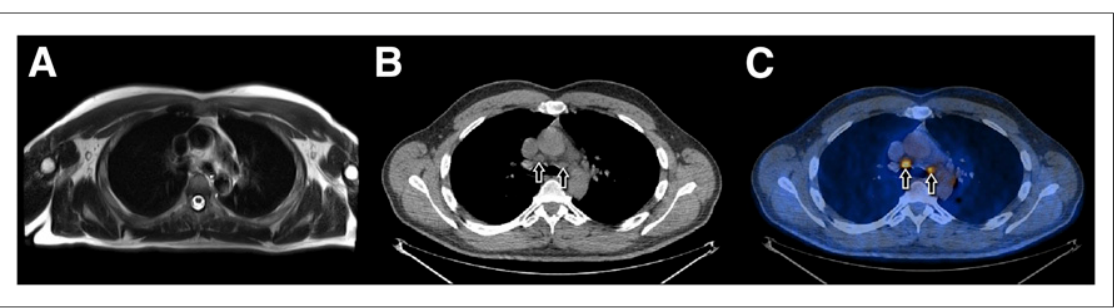

FIGURE 3. A 47-y-old male with lung cancer. (A) Axial T2WI HASTE image of chest at level of carina shows no obvious lymph nodes. Axial CT (B) and axial fused CT (C) images of chest at level of carina show 2 tracheobronchial lymph nodes (short arrows), which show tracer avidity. interobserver agreement in assigning traceravid lesions to an anatomic site are summarized in Table 2. The observers disagreed by 1 score category with respect to $4 \%$ of lesions for PET/CT and $1 \%$ of lesions for PET/MR imaging. There were no cases of disagreement by 2 categories. The intraclass correlation coefficient results indicate statistically significant agreement between observers in all cases $(P<0.01)$. The intraobserver agreement mean percentage for anatomic localization was $93 \%$ (Table 3). There was disagreement by 1 localization category was no significant difference $(P=0.025)$ in PET/CT and PET/MR image alignment. Minor misalignment was observed in 20 of 50 cases for PET/CT and 15 of 50 cases for PET/MR imaging, mostly in the head and neck region.

\section{Lesion Identification and Localization}

Two hundred twenty-seven tracer-avid lesions were identified across both modalities.

PET/CT. Of the 227 lesions, 225 were correctly identified on the PET component of PET/CT, whereas the remaining 2 lesions, which were around the bladder dome, were thought to represent bladder diverticula and were disregarded by all 3 observers. Of these 225 lesions, 211 were also seen on the CT component (either as nodules or structural change) of PET/CT whereas 14 lesions identified on PET showed no structural change on CT (prostate, 3; small liver metastases from neuroendocrine tumor, 3; leiomyosarcoma, 1; vaginal melanoma, 1 ; ovarian tumor, 1 ; cervical cancer, 1 ; squamous cell carcinoma of the tongue, 1; drop metastases at the cervicomedullary junction, 1; C6 nerve root, 1; and adductor muscle deposit, 1). These lesions were not visualized on the CT component because of poor soft-tissue contrast but were accurately anatomically localized by the MR imaging component of PET/MR imaging.

PET/MR Imaging. All 227 tracer-avid lesions were identified by the PET component of PET/MR imaging. The 2 lesions thought to be diverticula on PET/CT were anatomically localized to the bladder dome due to deposits from vaginal paraganglioma (Fig. 2). Thus, the percentage observer agreement between PET/CT and PET/MR lesion identification was $98.7 \%$. Of the 227 tracer-avid lesions, 224 were seen on the MR imaging component of PET/ MR imaging, whereas 3 lesions identified on PET were not seen on MR imaging (lung, 1; mediastinal lymph nodes, 2) (Fig. 3). These lesions were accurately localized by the CT component of PET/CT.

\section{Interobserver and Intraobserver Agreement Within \\ Each Modality}

The interobserver agreement mean percentage was $96 \%$ for $\mathrm{PET} / \mathrm{CT}$ and $99 \%$ for PET/MR imaging. The results regarding in $6.2 \%$ of cases and by 2 categories in $0.8 \%$. This disagreement in localization included cases in which PET/MR imaging improved localization, compared with PET/CT (i.e., pelvis, colorectal), and cases in which PET/CT localization was superior to PET/MR imaging (lung, mediastinum). Overall, PET/MR imaging improved confidence in anatomic localization by $5.1 \%$, compared with PET/CT.

The SUV-based analysis of the lesions showed significant $(P<$ $0.01)$ increase (10\% on average) in lesion mean SUVs measured in PET/MR imaging, compared with PET/CT (Table 4). However, in normal areas of brain, mediastinum, liver, and soft tissue, there was a reduction in SUV in the range of $6 \%-18 \%$ due to washout of activity (Fig. 4). A strong positive correlation between PET/CTand PET/MR imaging-derived SUVs was obtained for both the tumor and the background regions (Table 4). The lesion SUV was always higher on PET/MR imaging, which was acquired $80 \pm 28 \mathrm{~min}$ (mean $\pm \mathrm{SD}$ ) after PET/CT. The lesion-to-liver SUV ratio was also measured and showed a strong positive correlation (Fig. 5). The 2 points highlighted in red on the figure correspond to cases in which PET/MR imaging was performed more than $2 \mathrm{~h}$ after the PET/CT acquisition.

In 45 of 50 patients, there was concordance between PET/CT and PET/MR imaging findings. In 5 patients $(10 \%)$, there was change in $\mathrm{T}$ staging of the disease based on the MR imaging component of PET/MR imaging.

Of the 5 patients, 1 patient had the tumor upstaged on PET/MR imaging, and the patient was offered palliative treatment rather than undergoing extensive surgery. In 2 of the 5 patients, the tumor was downstaged and the treatment was changed from palliative to local surgery and radical radiotherapy. In 1 of the 5 patients, 2 additional lesions were identified over the dome of the bladder on PET/MR imaging, which was within the surgical field, that were missed on PET/CT, thought to be urinary bladder diverticula. In 1 lung cancer patient with mediastinal nodal disease, PET/CT proved to be better than PET/MR imaging in $\mathrm{T}$ and $\mathrm{N}$ staging. In the rest of the patients, there was no change in management.

\section{Clinical Impact}

TABLE 2

Interobserver Agreement in Assigning Tracer-Avid Lesions to Anatomic Site

\begin{tabular}{|c|c|c|c|c|c|c|}
\hline \multirow[b]{2}{*}{ Interobserver results } & \multicolumn{3}{|c|}{$\mathrm{PET} / \mathrm{CT}$} & \multicolumn{3}{|c|}{ PET/MR imaging } \\
\hline & $\begin{array}{l}\text { Agreement } \\
(\%)\end{array}$ & $\begin{array}{c}\text { Intraclass correlation } \\
\text { coefficients }\end{array}$ & $P$ & $\begin{array}{l}\text { Agreement } \\
\quad(\%)\end{array}$ & $\begin{array}{l}\text { Intraclass correlation } \\
\text { coefficients }\end{array}$ & $P$ \\
\hline Observer 1-observer 2 & 97 & 0.89 & $<0.01$ & 98 & 0.61 & $<0.01$ \\
\hline Observer $1-$ observer 3 & 96 & 0.69 & $<0.01$ & 99 & 0.87 & $<0.01$ \\
\hline Observer 2 -observer 3 & 96 & 0.76 & $<0.01$ & 99 & 0.73 & $<0.01$ \\
\hline
\end{tabular}


TABLE 3

Anatomic Lesion Localization for PET/CT and PET/MR Imaging

\begin{tabular}{cccc}
\hline & \multicolumn{3}{c}{ PET/CT to PET/MR } \\
\cline { 2 - 4 } Intraobserver & $\begin{array}{c}\text { Intraclass } \\
\text { correlation } \\
\text { results }\end{array}$ & $\begin{array}{c}\text { Agreement } \\
\text { coefficients }\end{array}$ & $P$ \\
\hline Observer 1 & 93 & 0.029 & 0.33 \\
Observer 2 & 93 & 0.045 & 0.25 \\
Observer 3 & 94 & 0.025 & 0.65 \\
\hline
\end{tabular}

\section{DISCUSSION}

This is the first U.K. study, to our knowledge, that compared observer agreement in detection and anatomic localization of lesions on integrated PET/MR imaging scanners and PET/CT and clinical impact on patient management.

Our results showed excellent interobserver and intraobserver agreement in lesion identification by the 3 readers for both modalities. Regarding lesion localization, the observers disagreed by 1 score category in $4 \%$ of cases for PET/CT and $1 \%$ of cases for PET/MR imaging.

Although the results indicate that most lesions could be accurately identified by either modality, lesions that had a low localization score on CT were well localized on T1WI, T2 HASTE, and DWI/ADC MR imaging and vice versa. Overall, PET/MR imaging improved confidence in anatomic localization by $5.1 \%$, compared with PET/CT; this was attributed to the established superior softtissue contrast seen in head and neck, pelvis, and colorectal cancer patients. In $1.3 \%$ of cases, CT identified more lesions, mostly in lung and mediastinal nodes. Thus, in difficult cases the 2 modalities complemented each other for lesion localization in specific anatomic territories where one or the other of the modalities is known to be superior.

Overall, the PET data on both modalities were of similar quality. The number of tracer-avid lesions detected by PET/CT and PET/MR imaging were similar, the only difference being that PET/MR imaging identified 2 additional lesions that on PET/CT were thought to be bladder diverticula. The image quality was slightly better for PET/CT, and alignment was better for PET/MR

TABLE 4

Mean SUV in Lesions and Background Regions on PET/CT and PET/MR Imaging

\begin{tabular}{|c|c|c|c|c|}
\hline \multirow[b]{2}{*}{ Site } & \multicolumn{2}{|c|}{ Mean SUV } & \multirow{2}{*}{\multicolumn{2}{|c|}{ Wilcoxon Spearman }} \\
\hline & $\begin{array}{c}\mathrm{PET} / \mathrm{CT} \\
\text { (mean } \pm \\
\mathrm{SD})\end{array}$ & $\begin{array}{c}\mathrm{PET} / \mathrm{MR} \\
\text { (mean } \pm \\
\mathrm{SD})\end{array}$ & & \\
\hline Tumor & $4.0 \pm 2.5$ & $4.4 \pm 2.4$ & $<0.01$ & 0.861 \\
\hline Liver & $3.5 \pm 3.1$ & $3.3 \pm 3.0$ & $<0.01$ & 0.883 \\
\hline Soft tissue & $0.7 \pm 0.3$ & $0.6 \pm 0.3$ & 0.23 & 0.46 \\
\hline Brain & $5.2 \pm 3.9$ & $4.5 \pm 3.9$ & $<0.01$ & 0.866 \\
\hline Mediastinum & $1.1 \pm 0.5$ & $0.9 \pm 0.5$ & $<0.01$ & 0.565 \\
\hline $\begin{array}{c}\text { Tumor/liver } \\
\text { SUVR }\end{array}$ & $1.9 \pm 1.4$ & $2.3 \pm 1.7$ & $<0.01$ & 0.913 \\
\hline
\end{tabular}

${ }^{*}$ SUVR $=$ SUV ratio.

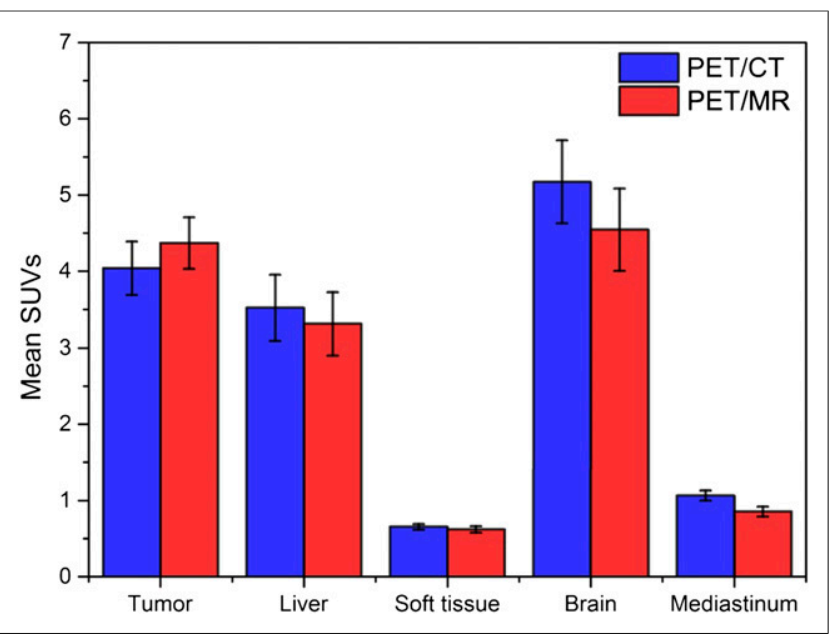

FIGURE 4. Mean SUVs for lesions and background regions.

imaging. The difference in image alignment was not statistically significant but was anticipated, because in PET/MR imaging the PET and MR images are acquired simultaneously for each bed position whereas for PET/CT the images are acquired sequentially (PET after CT). This temporal mismatch can cause image artifacts due to respiration motion, bowel movement, or patient movement between the 2 scans and has been reported previously (18). Our results show that the image quality had no significant influence on the interpretation of PET/CT or PET/MR imaging studies.

Regarding quantification, a systematic increase in PET/MR imaging SUVs in lesions was noted. The 2 modalities performed equally well in qualitative lesion detection, but, as expected, the lesion-tobackground contrast was more evident when the interval between ${ }^{18}$ F-FDG PET/CT and PET/MR imaging was longer because of increased accumulation within the lesion and washout from the background as has been reported previously (18-21). Similar findings were reported by Drezga et al. (18) regarding the lesion-tobackground SUV ratio, but in contrast to our study, they also found a decrease in lesion SUV. Such variances may be due to different methods used for SUV calibration of various manufacturers' systems but also importantly to different time intervals between the 2 scans because it is expected that the lesion SUV increases over time, and

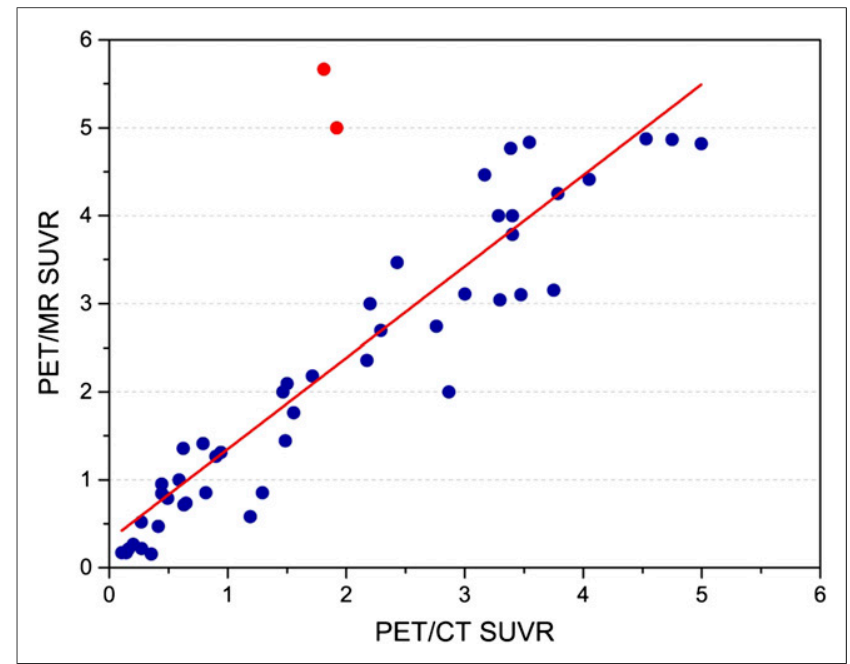

FIGURE 5. Comparison of lesion-to-liver ratio. SUVR = SUV ratio. 


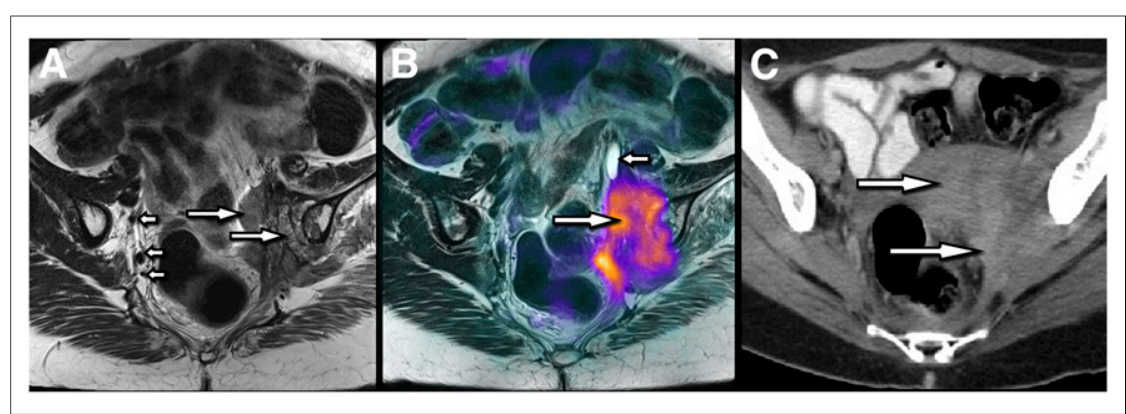

FIGURE 6. A 76-y-old woman with history of treated cervical cancer presented with suspected recurrence. (A) Axial oblique T2-weighted image of pelvis shows abnormal soft-tissue lesion (long arrows) involving left pelvic side wall and left iliac bone. This is when compared with other side (short arrow), shows perineural and vascular tumoral infiltration. (B) Fused ${ }^{18} \mathrm{~F}$-FDG PET and T2-weighted axial-oblique image shows ${ }^{18} \mathrm{~F}-\mathrm{FDG}$-avid soft-tissue tumor in left side of pelvis (long arrow) extending though sciatic notch with secondary hydoureter (short arrow) and involvement of left iliac bone. (C) Axial CT image of pelvis shows softtissue mass (long arrows) involving left-side wall of pelvis with no defined margins or clear neurovascular infiltration.

this has previously been exploited in dual-time point studies $(20,21)$.

It has been shown in previous studies that PET/MR imaging is superior to PET/CT in the investigation of local recurrent disease or residual disease in postoperative or postchemoradiotherapy patients, especially those with head and neck tumors (Fig. 6). Nakamoto et al. reported a sensitivity of $92 \%$ of PET/MR imaging for disease recurrence (22). However, they did not acquire the PET/MR imaging data simultaneously but fused the PET and MR imaging components.

In this study, PET/MR imaging showed a marginal improvement in confidence $(5.1 \%)$ in the anatomic localization of lesions. In $10 \%$ of patients, there was clear improvement in $\mathrm{T}$ staging on PET/MR imaging, where T2 HASTE and DWI were most useful and showed the degree of tumor extension into adjacent tissue because of the superior soft-tissue contrast and anatomic detail. It is speculated that any clinical impact will be driven by the MR imaging and CT components of PET/MR imaging and PET/CT and that newer PET radiopharmaceuticals and the addition of dynamic contrast-enhanced MR imaging, DWI, and MR spectroscopy will increase the sensitivity and specificity (23-25) and may also have a clinical impact. PET/CT is likely to remain the preferred imaging modality for lung and mediastinal nodal disease. However, with only 4 patients evaluated, our results were limited in assessing this tumor specifically. With advancing lung MR imaging sequences, PET/MR may soon develop into a more useful technique for thoracic imaging.

PET/MR imaging could be used for organ-specific imaging, which would allow $\mathrm{T}$ and $\mathrm{N}$ staging of site-specific disease such as prostate, liver, or brain tumors. However, in our study we opted for whole-body assessment, which offers the added benefit of $\mathrm{M}$ staging.

Some limitations of this study need to be mentioned. The patient number was relatively small. The number of each individual tumor subtype was small. In this study, patients were first scanned on the PET/CT system, and comparisons need to be conducted in which patients are instead first scanned with PET/ MR imaging. We did not evaluate the lesion size or the effects of partial volume in this study. In our study, unenhanced CT with low radiation dose was used; it is possible that the use of contrast-enhanced diagnostic CT as part of the PET/CT examination would have improved the performance of PET/CT versus PET/MR imaging. Overall, the MR imaging acquisition time was longer than the CT acquisition time, and it was not well tolerated by some patients. Finally, MR imaging contraindications and phobic patients precluded some studies.

\section{CONCLUSION}

This first, to our knowledge, pilot study has demonstrated the effectiveness of integrated PET/MR whole-body imaging in cancer and its clinical impact on patient's management in selected patients through a multidisciplinary approach. Comparison of the qualitative data acquired with conventional PET/CT and integrated PET/MR imaging revealed no significant differences, with good percentage inter- and intraobserver agreement in anatomic lesion localization across both modalities and significant correlation for quantitative results (mean SUV). PET data on both modalities were similar; however, the superior soft-tissue resolution of MR imaging over CT in cancers of the head and neck, pelvis, and colon or rectum was demonstrated. PET/CT is likely to remain the preferred imaging modality for lung and mediastinal nodal disease.

\section{DISCLOSURE}

The costs of publication of this article were defrayed in part by the payment of page charges. Therefore, and solely to indicate this fact, this article is hereby marked "advertisement" in accordance with 18 USC section 1734 . No potential conflict of interest relevant to this article was reported.

\section{REFERENCES}

1. Syed R, Bomanji JB, Nagabhushan N, et al. Impact of combined ${ }^{18} \mathrm{~F}-\mathrm{FDG}$ PET/ CT in head and neck tumors. Br J Cancer. 2005;92:1046-1050.

2. Townsend DW, Beyer T, Kinahan PE, et al. The SMART scanner: a combined PET/CT tomography for clinical oncology. Nucl Sci Symp, 1998. 1998;2:11701174 .

3. Pichler BJ, Wehrl HF, Judenhofer MS. Latest advances in molecular imaging instrumentation. J Nucl Med. 2008;49:5S-23S.

4. Collins CD. PET/CT in oncology: for which tumors is it the reference standard? Cancer Imaging. 2007(spec no A);7:77-83.

5. Bomanji JB, Costa DC, Ell PJ. Clinical role of positron emission tomography in oncology. Lancet Oncol. 2001;2:157-164.

6. Townsend DW. Dual-modality imaging: combining anatomy and function. J Nucl Med. 2008;49:938-955.

7. Antoch G, Bockisch A. Combined PET/MRI: a new dimension in whole-body oncology imaging? Eur J Nucl Med Mol Imaging. 2009;36:S113-S120.

8. Antoch G, Vogt FM, Freudenberg LS, et al. Whole-body dual-modality PET/CT and whole-body MRI for tumor staging in oncology. JAMA. 2003;290:31993206.

9. Müller-Horvat C, Radny P, Eigentler TK, et al. Prospective comparison of the impact on treatment decisions of whole-body magnetic resonance imaging and computed tomography in patients with metastatic malignant melanoma. Eur J Cancer. 2006;42:342-350. 
10. Smith AB, Dillon WP, Lau BC, et al. Radiation dose reduction strategy for CT protocols: successful implementation in neuroradiology section. Radiology. 2008;247:499-506.

11. Antypas EJ, Sokhandon F, Farah M, et al. A comprehensive approach to CT radiation dose reduction: one institution's experience. AJR. 2011;197:935-940.

12. Boellaard R. Standards for PET image acquisition and quantitative data analysis. J Nucl Med. 2009;50:11S-20S.

13. Teräs M, Tolvanen T, Johansson JJ, Williams JJ, Knuuti J. Performance of the new generation of whole-body PET/CT scanners: Discovery STE and Discovery VCT. Eur J Nucl Med Mol Imaging. 2007;34:1683-1692.

14. Martinez-Möller A, Souvatzoglou M, Delso G, et al. Tissue classification as a potential approach for attenuation correction in whole-body PET/MRI: evaluation with PET/CT data. J Nucl Med. 2009;50:520-526.

15. Samarin A, Burger C, Wollenweber S, et al. PET/MR Imaging of bone lesions: implications for PET quantification from imperfect attenuation correction. Eur J Nucl Med Mol Imaging. 2012;39:1154-1160.

16. Catana C, van der Kouwe A, Benner $\mathrm{T}$, et al. Toward implementing an MRI-based PET attenuation-correction method for neurologic studies on the MR-PET brain prototype. J Nucl Med. 2010;51:1431-1438.

17. Wagenknecht G, Kaiser H, Mottaghy F, Herzog H. MRI for attenuation correction in PET: methods and challenges. MAGMA. 2013;26:99-113.

18. Drzezga A, Souvatzoglou M, Eiber M, et al. First clinical experience with integrated whole-body PET/MRI: comparison to PET/CT in patients with oncologic diagnoses. J Nucl Med. 2012;53:845-855.
19. Paquet N, Albert A, Foidart J, Hustinx R. Within-patient variability of ${ }^{18} \mathrm{~F}-$ FDG: standardized uptake values in normal tissues. J Nucl Med. 2004;45: 784-788.

20. Lee JW, Kim S-K, Lee SM, Moon SH, Kim T-S. Detection of hepatic metastases using dual-time-point FDG PET/CT scans in patients with colorectal cancer. Mol Imaging Biol. 2011;13:565-572.

21. Matthies A, Hickeson M, Cuchiara A, Alavi A. Dual time point ${ }^{18}$ F-FDG PET for the evaluation of pulmonary nodules. J Nucl Med. 2002;43:871-875.

22. Nakamoto Y, Tamai K, Saga T, et al. Clinical value of image fusion from MR and PET in patients with head and neck cancer. Mol Imaging Biol. 2009;11: 46-53.

23. Laurent V, Trausch G, Bruot O, Oliver P, Felblinger J, Regent D. Comparative study of two whole-body imaging techniques in the case of melanoma metastases: advantages of multi-contrast MRI examination including a diffusionweighted sequences in comparison with PET/CT. Eur J Radiol. 2010;75: 376-383.

24. Khayal IS, McKnight TR, McGue C, et al. Apparent diffusion coefficient and fractional anisotropy of newly diagnosed grade II gliomas. NMR Biomed. 2009; 22:449-455.

25. McBride DQ, Miller BL, Nikas DL, et al. Analysis of brain tumors using ${ }^{1} \mathrm{H}$ magnetic resonance spectroscopy. Surg Neurol. 1995;44:137-144. 